\title{
About the special topic of mechanics and biomimetics of biomaterials and animal locomotion
}

\author{
Xi Qiao Feng · Mao Sun
}

Published online: 13 February 2010

(C) The Chinese Society of Theoretical and Applied Mechanics and Springer-Verlag GmbH 2010

Scientists have increasingly been turning their attentions to searching for inspirations from the splendid living world in order to solve various challenging problems of technologically importance. Rapid advances in observation techniques and computational ability will make it a reality to understand, at different time and space scales, the functions, structures and properties of natural materials as well as the complicated behaviors of swimming, flying and running of animals. In the last decades, much attentions have been attracted on the superior properties and striking abilities of biomaterials and animals.

Considering the rapid development and technological important applications of mechanics and biomimetics of biomaterials and animal locomotion, the International Workshop on Mechanics and Biomimetics of Biomaterials \& Animal Locomotion (IWMBAL-2008) was hold during December 9-12, 2008 at Sanya, China. This workshop was organized by the Chinese Society of Theoretical and Applied Mechanics (CSTAM) and International Center for Theoretical and Applied Mechanics in Beijing (BICTAM) and chaired by Professors Mao Sun and Xi-Qiao Feng.

The aim of this workshop was to join internationally famous experts to exchange and explore the recent developments in the study of mechanics and biomimetics of natural biomaterials and animal locomotions, a subject of great

\section{Q. Feng $(\varangle)$}

Institute of Biomechanics and Medical Engineering,

Department of Engineering Mechanics, Tsinghua University,

100084 Beijing, China

e-mail: fengxq@tsinghua.edu.cn

\section{Sun}

Institute of Fluid Mechanics, Beihang University,

100083 Beijing, China

e-mail: m.sun@263.net scientific interest. Its scope was focused on the following closely relevant issues:

(1) Functions, structures, properties and their relations of natural biomaterials,

(2) Multiscale mechanics and modeling of natural biomaterials,

(3) Biomimetic or bioinspired design and fabrications of novel materials,

(4) Biomechanics or bioinspired of animal flying and swimming,

(5) Biomechanics of animal terrestrial locomotion,

(6) Biomimetics of micro-sized air vehicles,

(7) Applications in such fields as medical engineering.

IWMBAL-2008 provided an international forum for presentations and discussions of the latest scientific and technological development in mechanics and biomimetics of biomaterials and animal locomotion. About 50 researchers from 9 countries/regions, including many internationally eminent scientists, participated in this academic event and delivered oral presentations.

In this issue of Acta Mechanica Sinica, we selected seven full papers published in IWMBAL-2008 after peer review. J. Cadman etal. examined the microstructures of cuttlebone, which possesses the multifunctional properties of high porosity, high flexural stiffness and compressive strength and, therefore, is a good example of design optimization of cellular structures created by nature. They reported an approximately periodic microstructure, appropriate for numerical characterization using direct homogenization techniques of micromechanics. Cadman et al. used the experimentally characterized results as the target values in an inverse homogenization procedure with the aim 
to regenerating microstructures with the same properties as cuttlebone. This study provides clues to develop biomimetic microstructural materials which share the same structures and properties as natural materials. J. Zhao et al. compared the mechanical properties and structure of Haliotis discus hannai Ino and Hemifusus tuba conch shells in order to comparatively investigate the mechanisms by which nature designs composites. The Hemifusus tuba conch shell possesses more refined and flexible microstructure than abalone shell, but the latter exhibits better bending strength. Therefore, the authors supposed that the size of inorganic substance is not a dominant factor to improve strength, and both the proteins in shells and the microstructure of inorganic phases play important roles.

Y.N. Wang and Q.H. Qin investigated the control mechanisms of mechanical bone remodeling at cellular level by means of an extensive parametric study. After analyzing all the combinations of 728 permutations in six model parameters, they identified a small number of parameter combinations that can lead to physiologically realistic responses which are similar to theoretically idealized physiological responses. This work is helpful to deep our understanding on mechanical bone remodeling and to develop combined pharmacological and mechanical therapies to treat bone loss diseases such as osteoporosis. X.F. Yao et al. experimentally studied the microstructures and properties of cancellous bone of avascular necrosis of femoral heads. They examined the differences between the steroid-injection group and the controlled group in order to investigate the property-microstructure relationship of cancellous bone.

Mechanical-electrical coupling effects are crucial for many biological functions and processes of cells, but there has been little theoretical investigation on electric effects on the deformation of cells. Using a recently established liquid crystal model for vesicles, L.T. Gao etal. presented a theoretical method to analyze the morphological transition of liquid crystal vesicles in an electric field. The coupled mechanical-electrical effects associated with elastic bending, osmotic pressure, surface tension, Maxwell pressure, as well as flexoelectric and dielectric properties of the membrane were considered. This study is helpful for understanding and revealing the morphological evolution mechanisms of vesicles in electric fields and some associated phenomena of cells.

As an attractive topic of mechanics and biomimetics, the mechanics of fish swimming and insect flying also attracted much attention during IWMBAL-2008. C.J. Wu and L. Wang investigated self-propelled swimming of two- and threedimensional biomimetic fish school in a viscous flow by using a computational fluid dynamics method. They found that due to the interactions of vortices in the wakes, a fish school swim with a given flapping rule could not keep the fixed shape of a queue if there was no proper control. Therefore, they proposed that the locomotion speed is adjusted by the flapping frequency of the caudal, and the direction of swimming is controlled by the swinging of the head of a fish. This novel control strategy is of great interest for construction of biomimetic robot fishes with robust control ability. $\mathrm{G}$. $\mathrm{Wu}$ and L.J. Zeng reported a two-dimensional fringe projection method to measure the kinematics of a free-flying hawk-moth (Macroglossum stellatarum). By calculating the three-dimensional coordinates of the points on the hawk-moth and reconstruction of the wing, they obtained the flight trajectory, body attitude and wing kinematics including flapping angle, elevation angle, torsion angle, and camber deformation.

We wish to thank all the authors and speakers who contributed papers and presentations to IWMBAL-2008. The supports from the National Natural Science Foundation of China (NSFC), CSTAM and BICTAM are acknowledged gratefully. We also thank the editors of Acta Mechanica Sinica for their support in publishing these papers. 